\title{
ORDER POLYNOMIALLY COMPLETE LATTICES MUST BE LARGE
}

\author{
MARTIN GOLDSTERN AND SAHARON SHELAH
}

\begin{abstract}
If $L$ is an o.p.c. (order polynomially complete) lattice, then the cardinality of $L$ is a strongly inaccessible cardinal. In particular, the existence of o.p.c. lattices is not provable in ZFC, not even from $\mathrm{ZFC}+\mathrm{GCH}$.
\end{abstract}

\section{INTRODUCTION}

Let $\left(L_{1}, \leq\right)$ and $\left(L_{2}, \leq\right)$ be partial orders. We call a map $f: L_{1} \rightarrow L_{2}$ "monotone" if $x \leq y$ implies $f(x) \leq f(y)$.

Notice that if $(L, \wedge, \vee, \leq)$ is a lattice, then every polynomial function (i.e., function induced by a lattice-theoretic polynomial) is monotone. We call a lattice $L n$-order polynomially complete (o.p.c.) if

$(*)$ every monotone $n$-ary function is a polynomial function and we say that $L$ is order polynomially complete if $L$ is $n$-order polynomially complete for every $n$.

The question whether there can be an infinite o.p.c. lattice has been the subject of several papers. Kaiser and Sauer [?] remarked that such a lattice cannot be countable, and Haviar and Ploščica showed in [?]

Date: July 4, 1997.

1991 Mathematics Subject Classification. Primary 06A07; secondary 08A40, 06B99, 03E55.

Key words and phrases. polynomially complete, inaccessible cardinal, canonization.

The first author is supported by an Erwin Schrödinger fellowship from the Austrian Science Foundation.

The second author is supported by the German-Israeli Foundation for Scientific Research \& Development Grant No. G-294.081.06/93. Publication number 633. 
that such a lattice would have to be at least of size $\beth_{\omega}$. (Here, $\beth_{0}=\aleph_{0}$, $\beth_{n+1}=2^{\beth_{n}}, \beth_{\omega}=\sup \left\{\beth_{n}: n \in \omega\right\}$.

We show here that the size of an infinite o.p.c. lattice (if one exists at all) must be a strongly inaccessible cardinal.

In particular, the existence of such a lattice cannot be derived from the "usual" axioms of mathematics, as codified in the Zermelo-Fraenkel axioms for set theory. Moreover, also certain additional assumptions such as the (generalized) continuum hypothesis are not sufficient to prove the existence of an o.p.c. lattice, or in other words, the theory $\mathrm{ZFC}+\mathrm{GCH}+$ "there is no infinite o.p.c. lattice" is consistent円. In fact, the well-known consistent ${ }^{1}$ theory $\mathrm{ZFC}+$ "there is no inaccessible cardinal", a natural extension of ZFC, proves that there is no o.p.c. lattice.

We still do not know whether the existence of an o.p.c. lattice can be refuted in ZFC alone.

In [?] we showed that if we change the original question by relaxing "lattice" to "partial order", and "polynomial" to "definable", then we get (consistently) a positive answer, already for a partial order of size $\aleph_{1}$.

Acknowledgement. We are grateful to Lutz Heindorf for his thoughtful comments on an earlier version of this paper, and for alerting us to $[?]$.

\section{Preliminaries}

We define here some of the notation that we will use, and we quote several well-known theorems and corollaries from the calculus of partitions.

1.1. Definition. We fix a set $\mathbf{R}=\{\langle\rangle,,=, \|\}$ of 4 symbols. For any p.o. $(L, \leq)$ we define $R: L \times L \rightarrow \mathbf{R}$ in the obvious way: $R(x, y)=<$ iff $x<y$, etc.

1.2. Definition. Let $(L, \leq)$ be a partial order.

\footnotetext{
${ }^{1}$ Pedants are invited to insert the necessary disclaimer themselves.
} 
(1) We say that a set $A \subseteq L$ is "co-well-ordered" iff $(A, \geq)$ is a well-ordered set.

(2) We call a set $A \subseteq L$ "uniform" iff $A$ is either an antichain, or a well-ordered chain, or a co-well-ordered chain.

(3) If $A$ is well-ordered we say that the type of $A$ is " $<$ ". Similarly we define the types " $>$ " and " $\|$ ". [This notation is ambiguous if $A$ is finite. However, we are mainly interested in (large) infinite sets anyway, so this ambiguity will not cause any problems.]

(4) Let $A_{i} \subseteq L$ for $i \in I$. We call $\left(A_{i}: i \in I\right)$ "canonical" if the following conditions hold:

(a) each $A_{i}$ is uniform, all $A_{i}$ are of the same type,

(b) there is a function $F: I \times I \rightarrow \mathbf{R}$ such that:

$$
\forall i, j \in I: i \neq j \Rightarrow \forall a \in A_{i} \forall b \in A_{j}: R(a, b)=F(i, j) .
$$

Loosely speaking, this says that whenever $i \neq j$, then either $A_{i}$ lies "completely above" $A_{j}$, or conversely, or $A_{i}$ is "completely incomparable" with $A_{j}$.

1.3. Definition. For any set $A,[A]^{2}$ is the set of unordered pairs from A:

$$
[A]^{2}=\{\{x, y\}: x, y \in A, x \neq y\}
$$

When we consider a set $A$ together with an 1-1 enumeration $A=\left\{a_{i}\right.$ : $i<\kappa\}$, we usually identify the unordered pair $\left\{a_{i}, a_{j}\right\} \in[A]^{2}$ with the ordered pair $\left(a_{i}, a_{j}\right)$ whenever $i<j$.

1.4. Definition. Let $\kappa, \lambda$ and $c$ be cardinals. The "partition symbol"

$$
\lambda \rightarrow(\kappa)_{c}^{2}
$$

means: Whenever $F:[L]^{2} \rightarrow C$, where $|L|=\lambda,|C|=c$, then there is an " $F$-homogeneous set" $K \subseteq L$ of cardinality $\kappa$, i.e., a set $K$ such that $F \uparrow[K]^{2}$ is constant. 
(In other words, whenever the edges of the complete graph on $\lambda$ many vertices are colored with $c$ colors, then there is a complete subgraph with $\kappa$ many vertices all of whose edges have the same color.)

1.5. Definition. Let $(L, \leq)$ be a partial order. We will try to get some information on the structure of $L$ by considering certain "cardinal characteristics" $\mu(L)$ and $\nu(L)$, which are defined as follows:

(1) We let $\mu(L)$ be the smallest cardinal $\mu$ such that there is no uniform set $A \subseteq L$ of cardinality $\mu$. In other words, $\kappa<\mu(L)$ iff there is a uniform subset $A \subseteq L$ of size $\kappa$.

(2) We let $\mu_{n}(L)=\mu\left(L^{n}\right)$ for $n>0$.

(3) We let $\nu(L)$ be the smallest cardinal $\nu$ such that there is no family $\left(f_{i}: i<\nu\right)$ of $\nu$ many pairwise incomparable monotone functions from $L$ to $L$. (Functions are ordered pointwise.)

(4) $\nu_{n}(L)$ is the smallest cardinal $\nu$ such that there are no pairwise incomparable monotone functions $\left(f_{i}: i<\nu\right)$ from $L^{n}$ to $L$.

(5) More generally, $\nu\left(L_{1}, L_{2}\right)$ is the the smallest cardinal $\nu$ such that there are no pairwise incomparable monotone functions $\left(f_{i}: i<\right.$ v) from $L_{1}$ to $L_{2}$.

(6) $\mu_{\infty}=\sup \left\{\mu_{n}: n \in \omega\right\}, \nu_{\infty}=\sup \left\{\nu_{n}: n \in \omega\right\}$. (Note that trivially $\mu_{n} \leq \mu_{n+1}$ and $\nu_{n} \leq \nu_{n+1}$ for all $n \in \omega$.)

1.6. Fact. Let $L$ be infinite. Then $\mu_{n}(L) \leq|L|^{+}$and $\nu(L) \leq\left(2^{|L|}\right)^{+}$.

1.7. Theorem (Ramsey). For any natural number $k, \aleph_{0} \rightarrow\left(\aleph_{0}\right)_{k}^{2}$.

Proof. See [?, 10.2].

1.8. Theorem (Erdös+Rado). For any infinite $\kappa,\left(2^{\kappa}\right)^{+} \rightarrow\left(\kappa^{+}\right)_{\kappa}^{2}$. Proof. See [?, 17.11(32)].

1.9. Theorem (Erdős+Rado). (1) If $\kappa$ is an infinite cardinal, $k$ finite, then $\left(2^{<\kappa}\right)^{+} \rightarrow(\kappa)_{k}^{2}$. 
ORDER POLYNOMIALLY COMPLETE LATTICES MUST BE LARGE

(2) If $\kappa$ is a strong limit cardinal, then

$$
\kappa^{+} \rightarrow(\kappa)_{4}^{2}
$$

Proof. [?, 15.2] proves a theorem that is stronger than (1). (2) is a special case of $(1)$.

1.10. Corollary. Let $(L, \leq)$ be a partial order.

(a) If $\kappa$ is an infinite cardinal, $|L|>2^{\kappa}$, then $\mu(L)>\kappa$. (In fact, $\mu(L)>\kappa^{+}$.)

(a') $|L| \leq 2^{\mu(L)}$.

(b) If $L$ is infinite, then $\mu(L)>\aleph_{0}$.

(c) If $\kappa$ is a strong limit cardinal, then $\kappa \leq \mu(L)$ iff $\kappa \leq|L|$.

(d) If $\kappa$ is a strong limit cardinal, then $|L|>\kappa$ implies $\mu(L)>\kappa$,

(e) If $\kappa$ is a strong limit cardinal, then $\mu(L)=\kappa$ implies $|L|=\kappa$.

Proof. (a) Write $\rho$ for $\left(2^{\kappa}\right)^{+}$. Let $\left(a_{i}: i<\rho\right)$ be distinct elements of $L$, and define $F:[\rho]^{2} \rightarrow \mathbf{R}$ by requiring $F(i, j)=R\left(a_{i}, a_{j}\right)$ whenever $i<j$. The Erdős-Rado theorem 1.8 promises us an $F$-homogeneous set $\left\{i_{\zeta}: \zeta<\kappa^{+}\right\}$of size $\kappa^{+}$, which will naturally induce a uniform set $\left\{a_{i_{\zeta}}: \zeta<\kappa^{+}\right\}$of the same cardinality.

(a') follows from (a).

The proofs of (b) and (d) are similar, using 1.7 and 1.9, respectively, instead of 1.8 .

(c) follows easily from 1.6. (e) follows from (c) and (d).

1.11. Canonization Theorem (Erdős+Hajnal+Rado). Let $\lambda$ be an infinite cardinal, $\left(A_{i}: i<\lambda\right)$ be a family of pairwise disjoint sets. Let $\left(\kappa_{i}: i<\lambda\right)$ be infinite cardinals satisfying $2^{\kappa_{i}}<2^{\kappa_{j}}$ whenever $i<j$, and assume $\left|A_{i}\right|>2^{\kappa_{i}}$. Let $f$ be a function with domain $[A]^{2}$, where $A=\bigcup_{i<\lambda} A_{i}$, and let the range of $f$ be small (say: finite). Moreover, assume $\kappa_{0} \geq 2^{\lambda}$.

For $\alpha \in A$ write $i_{\alpha}$ for the unique $i$ such that $\alpha \in A_{i}$. 
Then there are sets $\left(A_{i}^{\prime}: i<\lambda\right),\left|A_{i}^{\prime}\right|=\kappa_{i}, A_{i}^{\prime} \subseteq A_{i}$, such that for $\alpha, \beta \in A^{\prime}\left(:=\bigcup_{i<\lambda} A_{i}^{\prime}\right), f(\alpha, \beta)$ depends only on $i_{\alpha}$ and $i_{\beta}$.

That is, there is a function $F$ with domain $[\lambda]^{2} \cup \lambda$ such that for all $\alpha \neq \beta$ in $A^{\prime}, f(\alpha, \beta)=F\left(i_{\alpha}, i_{\beta}\right)$.

Proof. See [?, 28.1].

1.12. Corollary. Let $\lambda=c f(\kappa)<\kappa, \kappa$ a strong limit cardinal, $|L|=$ $\kappa,(L, \leq)$ a partial order. Then there is is a family $\left(A_{\zeta}: \zeta<\lambda\right)$ of subsets of $L$ satisfying

(1) $\left(A_{\zeta}: \zeta<\lambda\right)$ is canonical. (See 1.9(4).)

(2) The sequence $\left(\left|A_{\zeta}\right|: \zeta<\lambda\right)$ is strictly increasing.

(3) $\sup \left\{\left|A_{\zeta}\right|: \zeta<\lambda\right\}=\kappa$.

(4) For all $\zeta<\lambda:\left|A_{\zeta}\right|>\lambda$.

Proof. Find an increasing sequence $\left(\kappa_{i}: i<\lambda\right)$ of cardinals such that $2^{\kappa_{i}}<\kappa_{i+1}$ for all $i$. Let $\left(a_{\alpha}: \alpha<\kappa\right)$ be distinct elements, and let $A_{i}:=\left\{a_{\alpha}: \kappa_{i+3}<\alpha<\kappa_{i+4}\right\}$ for $i<\lambda$. Thus, $A:=\bigcup_{i<\lambda} A_{i}$ is a disjoint union, and $\left|A_{i}\right|=\kappa_{i+4}>2^{\kappa_{i}}$. (Note that in this enumeration each set $A_{i}$ comes "before" $A_{j}$ for $i<j$.)

Define $f:[A]^{2} \rightarrow \mathbf{R}$ by letting $f\left(\left\{a_{\alpha}, a_{\beta}\right\}\right)=R\left(a_{\alpha}, a_{\beta}\right)$ for $\alpha<\beta$, and apply the canonization theorem 1.11.

The resulting sets $\left(A_{i}^{\prime}: i<\lambda\right)$ will be canonical.

1.13. Remark. If $\left(\kappa_{i}: i<\lambda\right)$ is increasing with limit $\kappa$, then $\prod_{i} \kappa_{i}=$ $\kappa^{\lambda}$. If moreover (as in our case) $\kappa$ is a strong limit cardinal, then $\kappa^{\lambda}=2^{\kappa}$.

Proof. See $[?, 6.4]$

\section{Partial maps}

We want to give lower estimates for $\nu(L)$, and then translate them to lower estimates for $\mu(L)$. (See 1.5 for the definitions of $\mu$ and $\nu$.) 
ORDER POLYNOMIALLY COMPLETE LATTICES MUST BE LARGE 7

Since we will typically only construct many partial functions that are pairwise incomparable, we have to give a sufficient condition that allows us to extend partial monotone functions to total monotone functions.

2.1. Fact. Let $L_{1}, L_{2}$ be partial orders. If $f: L_{1} \rightarrow L_{2}$ is a partial monotone function whose range is contained in a complete partial order $L_{2}^{\prime} \subseteq L_{2}$, then $f$ can be extended to a total monotone function $\hat{f}: L_{1} \rightarrow$ $L_{2}$.

Proof. Let $\hat{f}(x)=\sup _{L_{2}^{\prime}}\{f(y): y \in \operatorname{dom}(f), y \leq x\}$.

2.2. Corollary. Let $L$ be a partial order, $\bar{A} \subseteq L$ a complete partial order. (Note: We only require that least upper bounds exist in $(\bar{A}, \leq)$, we do not care if these bounds are also least upper bounds in L.)

Then for any $A \subseteq \bar{A}$ we have $\nu(L) \geq \nu(A)$.

Proof. Every monotone map $f: A \rightarrow A$ can be extended to a monotone map $\hat{f}: L \rightarrow \bar{A}$. If $f, g$ are incomparable, then so are $\hat{f}, \hat{g}$.

So we will show that $\nu(L)$ is large by showing that $\nu(A)$ is large, for some sufficiently "nice" $A$.

In our treatment, "nice" means in particular "complete" (as a partial order), or at least "contained in a complete p.o." Here the following lemma, due to Kaiser and Sauer [?] will be helpful:

2.3. Lemma. If $(L, \leq)$ is an o.p.c. lattice, then $L$ is bounded (i.e., has a greatest and a smallest element).

Proof. See [?].

Our method to make $\nu(A)$ large will be multiplication: If $A_{1}, A_{2}, \ldots$ are sufficiently "independent" (in a sense to be made precise below), and $f_{i}: A_{i} \rightarrow A_{i}$ are monotone, then we will show that they can be combined to give a monotone function from $A:=\bigcup_{i} A_{i}$ to $A$.

2.4. Independence Lemma. Let $L$ be a partial order, $A=\bigcup_{i<\lambda} A_{i} \subseteq$ $L$ and assume that $\left(A_{i}: i<\lambda\right)$ is canonical. Then: 
(1) Whenever $\left(f_{i}: i<\lambda\right)$ is a family of functions, each $f_{i}: A_{i} \rightarrow A_{i}$ monotone, then the function $f:=\bigcup_{i<\lambda} f_{i}$ is a monotone function from $A$ to $A$.

(2) If $B$ is a partial order, $\left(f_{i}: i<\lambda\right)$ is a family of functions, each $f_{i}: A_{i} \rightarrow B$ monotone, and if $\bigcup_{i} A_{i}$ is an antichain, then $\bigcup_{i} f_{i}$ is monotone from $A$ to $B$.

(3) Moreover, if $\left(f_{i}: i<\lambda\right)$ and $\left(f_{i}^{\prime}: i<\lambda\right)$ are both as in (1) or (2), and for some $j$ the functions $f_{j}$ and $f_{j}^{\prime}$ are incomparable, then also $\bigcup_{i} f_{i}$ and $\bigcup_{i} f_{i}^{\prime}$ are incomparable.

(4) If $\kappa_{i}<\nu\left(A_{i}\right)$ for $i<\lambda$, then $\nu(A)>\prod_{i<\lambda} \kappa_{i}$.

(5) If $\kappa_{i}<\nu\left(A_{i}, B\right)$ for $i<\lambda$, and $A=\bigcup_{i<\lambda} A_{i}$ is an antichain, then $\nu(A, B)>\prod_{i<\lambda} \kappa_{i}$ (where $B$ is an arbitrary partial order)

Proof. (1) Let $F: I \times I \rightarrow \mathbf{R}$ witness that $\left(A_{i}: i \in I\right)$ is canonical. To check that $f$ is monotone, consider an arbitrary pair $a \leq b$ in $A$.

Now either there is a single $i$ with $a, b \in A_{i}$ then $f(a) \leq f(b)$ (because $f\left\lceil A_{i}=f_{i}\right.$ is monotone), or we have $i \neq j, a \in A_{i}, b \in A_{j}$. But then we must have $F(i, j)=<$, so (since $f(a) \in A_{i}, f(b) \in A_{j}$ ), we again have $f(a) \leq f(b)$.

(2) and (3) are easy.

(4) follows from (1) and (3), and (5) follows from (2) and (3).

Now that we know how to get pairwise incomparable functions by multiplication, we have to look more closely at the factors in this product. The factors are of the form $\nu(A)$, where $A$ is a uniform set. The computation of this cardinal characteristic turns out to be easy:

2.5. Fact. (a) If $A$ uniform, $|A|>2$, then $\nu(A)>2$.

(b) If $A$ is uniform, $|A|=\kappa \geq \aleph_{0}$, then $\nu(A)>2^{\kappa}$, i.e., there are $2^{\kappa}$ many pairwise incomparable monotone functions from $A$ to $A$.

(c) If $A$ is an antichain, $|A|=\kappa \geq \aleph_{0}$, then $2^{\kappa}<\nu(A,\{0,1\})$, i.e., there are $2^{\kappa}$ many incomparable (necessarily monotone) functions from $A$ into the two-element lattice $\{0,1\}$.

Proof. (a) Left to the reader. 
ORDER POLYNOMIALLY COMPLETE LATTICES MUST BE LARGE

(b) This is is trivial if $A$ is an antichain. So wlog assume that $A$ is well-ordered. Write $A$ as a union of $\kappa$ many disjoint convex sets $\bigcup_{i<\kappa} A_{i}$, each $A_{i}$ of cardinality $>2$. Then $\left(A_{i}: i \in I\right)$ is canonical. So we can apply (a) and the independence lemma 2.4(4), and get $\nu(A)>$ $\prod_{i<\kappa} 2=2^{\kappa}$.

(c): Let $A=\bigcup_{i} A_{i}$, where each $A_{i}$ is of size $>2$, and the $A_{i}$ are pairwise disjoint. Clearly $\nu\left(A_{i},\{0,1\}\right)>2$, so by the independence lemma 2.4 $\nu(A,\{0,1\})>2^{\kappa}$.

\section{3. $\mu$ AND $\nu$}

We now turn our attention to the number $\mu_{n}(L)$.

3.1. Fact. If $A \subseteq L^{n}$ is well-ordered of order type $\kappa$, then there is $A^{\prime} \subseteq L$, also well-ordered of order type $\kappa$.

Proof. Let $\bar{a}^{i}=\left(a^{i}(1), \ldots, a^{i}(n)\right)$ for $i<\kappa$, and $i<j \Rightarrow \bar{a}^{i}<\bar{a}^{j}$. For each $k \in\{1, \ldots, n\}$ the sequence $\left(a^{i}(k): i<\kappa\right)$ is weakly increasing. If the sequence $\left(a^{i}(k): i<\kappa\right)$ does not contain a strictly increasing sequence of length $\kappa$, then it must be eventually constant. However, this cannot happen for every $k \in\{1, \ldots, n\}$.

Now we finally investigate the relation between $\mu$ and $\nu$. It turns out to be slightly simpler if we look at $\mu_{\infty}$ and $\nu_{\infty}$ first.

First we show in 3.2 that the existence of many incomparable monotone functions from $L^{n}$ to $L\left(\kappa<\nu_{n}(L)\right)$ implies the existence of a large antichain in some $L^{m}\left(\kappa<\mu_{m}(L)\right)$, assuming that $L$ is o.p.c. (This is actually the only place in the whole proof where we talk about lattices rather than general partial orders.)

Then we show in 3.3 that a large (anti)chain in $L^{m}\left(\kappa<\mu_{m}(L)\right)$ implies the existence of $*$ very $*$ many incomparable monotone functions from $L^{m}$ to $L\left(2^{\kappa}<\nu_{m}(L)\right)$.

These two lemmata are (with minor modifications) taken from [?]. 
Finally in 3.4 we combine 3.2 and 3.3 to show that $\mu=\mu_{\infty}$ must be a strong limit cardinal.

3.2. Lemma. Let $(L, \leq)$ be an o.p.c. lattice, $\kappa$ a cardinal of uncountable cofinality. If $\kappa<\nu_{n}(L)$, then $\kappa<\mu_{\infty}(L)$. In particular:

(A) $\nu_{\infty} \leq \mu_{\infty}^{+}$

(B) $\nu_{\infty} \leq \mu_{\infty}$, if $\mu_{\infty}$ has uncountable cofinality.

Proof. Assume $\kappa<\nu_{n}(L)$. Let $\left(f_{i}: i<\kappa\right)$ be pairwise incomparable functions from $L^{n}$ to $L$. Since $L$ is o.p.c., each of these functions is a polynomial function. Thus, for each $i$ there is some natural number $k_{i}$ and a lattice-theoretic term $t_{i}\left(x_{1}, \ldots, x_{n}, y_{1}, \ldots, y_{k_{i}}\right)$ and a $k_{i}$-tuple $\bar{b}^{i}=\left(b_{1}^{i}, \ldots, b_{k_{i}}^{i}\right)$ such that for all $a_{1}, \ldots, a_{n}$ we have $f_{i}\left(a_{1}, \ldots, a_{n}\right)=$ $t_{i}\left(a_{1}, \ldots, a_{n}, b_{1}, \ldots, b_{k_{i}}\right)$.

Since there are only countably many pairs $\left(t_{i}, k_{i}\right)$ and we have assumed $c f(\kappa)>\aleph_{0}$, we may assume that they all are equal, say to $\left(t^{*}, k^{*}\right)$. But then $\left(\bar{b}^{i}: i<\kappa\right)$ must be pairwise incomparable in $L^{k^{*}}$, because $\bar{b}^{i} \leq \bar{b}^{j}$ would imply $f_{i} \leq f_{j}$. Hence we have found an antichain of size $\kappa$ in $L^{k^{*}}$.

To get $(\mathrm{A})$, let $\kappa:=\mu_{\infty}^{+}$, so $c f(\kappa)>\aleph_{0}$ and therefore " $\kappa<\nu_{\infty}$ " is impossible. To get (B), let $\kappa=\mu_{\infty}$.

From now on we can forget about lattices as long as we only consider partial orders having properties 3.2(A) and 3.2(B).

3.3. Lemma. Let $(L, \leq, 0,1)$ be a bounded partial order, $\kappa$ an infinite cardinal. If $\kappa<\mu_{n}(L)$, then $2^{\kappa}<\nu_{n}(L)$. In particular, $\kappa<\mu_{\infty}$ implies $2^{\kappa}<\nu_{\infty}$

Proof. Let $A \subseteq L^{n}$ be uniform of size $\kappa$.

Case 1: $A$ is a chain, so by 3.1 wlog $n=1$. Let $\bar{A}=A \cup\{0,1\}$. By fact 2.5, $\nu(A)>2^{\kappa}$. Since $\bar{A}$ is a complete partial order, we may apply fact 2.1 to get $\nu(L) \geq \nu(A)$. Hence $\nu(L)>2^{\kappa}$.

Case 2: $A$ is an antichain. Use 2.5(c). 
3.4. Conclusion. If $L$ is infinite and o.p.c., (or, slightly more generally, if $L$ is an infinite bounded partial order satisfying the conclusion of 3.9), then

(a) $\mu_{\infty}(L)$ must be a strong limit cardinal,

(b) $\mu(L)=\mu_{\infty}(L)$

(c) $|L|=\mu(L)$.

(d) $\nu(L)=|L|$.

Proof. (a) If $\kappa<\mu_{\infty}(L)$, then $2^{\kappa}<\nu_{\infty}(L)$ by 3.3. Now $2^{\kappa}$ always has uncountable cofinality, so we get $2^{\kappa}<\mu_{\infty}(L)$ by 3.2 .

(b) Assume that $\mu(L)<\mu_{\infty}(L)$. Let $\lambda=2^{2^{\mu(L)}}<\mu_{\infty}(L)$. By 1.10, $|L| \leq 2^{\mu(L)}<\lambda$, so $\mu_{n}(L) \leq|L|^{+} \leq \lambda$ for all $n \in \omega$, hence $\mu_{\infty}(L) \leq \lambda$, a contradiction.

(c) Use $1.10(\mathrm{e})$.

(d) $3.2\left(\right.$ B) implies $\nu(L) \leq \nu_{\infty}(L) \leq \mu(L)$, and 3.3 implies $\mu_{1}(L) \leq$ $\nu_{1}(L)$.

\section{THE MAIN LEMMA}

We have already shown that for an o.p.c. lattice $L$ the cardinal characteristic $\mu(L)$ must be a strong limit cardinal. In this section we show that $\mu(L)$ must be regular.

Letting $\kappa:=\mu(L)$ we first show that the singularity of $\kappa$ would imply the existence of $\gg \kappa$ many incomparable monotone functions, and then show that this would imply $\mu(L)>\kappa$.

4.1. Main Lemma. Let $(L, \leq, 0,1)$ be a bounded partial order, and let $\kappa$ be a singular strong limit cardinal, $\kappa \leq|L|$.

Then $\nu(L)>\kappa$.

If moreover $\operatorname{cf}(\kappa)=\aleph_{0}$, then we get even $\nu(L)>2^{\kappa}$. 
Proof. Let $\lambda=c f(\kappa)$. The first step in the proof of 4.1 is to find a canonical family $\left(A_{i}: i<\lambda\right)$ which is large, i.e., $\left|\bigcup_{i} A_{i}\right|=\kappa$, and $\prod_{i<\lambda}\left|A_{i}\right|=\kappa^{\lambda}=2^{\kappa}$. If the set $A:=\bigcup_{i} A_{i}$ happens to be a chain or antichain, we easily get $2^{\kappa}$ many pairwise incomparable monotone functions. Using the independence lemma we will show that already the canonicity of $A$ is sufficient to get many monotone functions.

In step 3 we will exhibit many pairwise incomparable (partial) functions from $A$ to $A$, so in step 2 we may have to massage $\left(A_{i}: i<\lambda\right)$ a bit to guarantee that these functions can be extended to total functions on $L$.

What actually happens in steps 2 and 3 depends on whether $\lambda$ is countable or not.

Step 1. Let $A=\left\{a_{i}: i<\kappa\right\}$ be distinct elements of our partial order. For $i<j<\kappa$ let $f(i, j)=R\left(a_{i}, a_{j}\right)$. (Recall 1.1.)

By the canonization theorem (or rather, by its corollary 1.12) we may (after thinning out our set $A$ ) wlog assume that $A=\bigcup_{\zeta<\lambda} A_{\zeta}$, where the cardinalities $\left|A_{\zeta}\right|$ are increasing with supremum $\kappa, \lambda<\left|A_{\zeta}\right|<\kappa$, and $\left(A_{\zeta}: \zeta<\lambda\right)$ is canonical. Let $\xi: \kappa \rightarrow \lambda$ be such that for all $i<\kappa, a_{i} \in A_{\xi_{i}}$. So there is a function $\left.F:[\lambda] \leq 2 \rightarrow\{<\rangle,, \|\right\}$ such that $R\left(a_{i}, a_{j}\right)=F\left(\left\{\xi_{i}, \xi_{j}\right\}\right)$ for all $i<j<\kappa$.

We may assume $\{0,1\} \cap A=\emptyset$. Let $\bar{A}=A \cup\{0,1\}$.

Note that

$$
\prod_{\xi<\lambda}\left|A_{\xi}\right|=\kappa^{c f(\kappa)}=2^{\kappa}
$$

Step 2, case a. Let us asume $\lambda=\aleph_{0}$ for the moment. So we have a canonical sequence $\left(A_{n}: n \in \omega\right)$, witnessed by $F:[\omega]^{2} \rightarrow\{<,>, \|\}$. By Ramsey's theorem 1.7 there is an infinite set $X \subseteq \omega$ such that $F$ is constant on $[X]^{2}$. By dropping some elements of the sequence $\left(A_{n}: n \in \omega\right)$ [i.e., replacing $\left(A_{n}: n \in \omega\right)$ by $\left(A_{n}: n \in X\right)$, and then for notational simplicity only pretending that $X=\omega]$ we may assume that $F$ is constant, say

$$
\forall n \forall k: n<k \Rightarrow \forall a \in A_{n} \forall b \in A_{k}: R(a, b)=c
$$




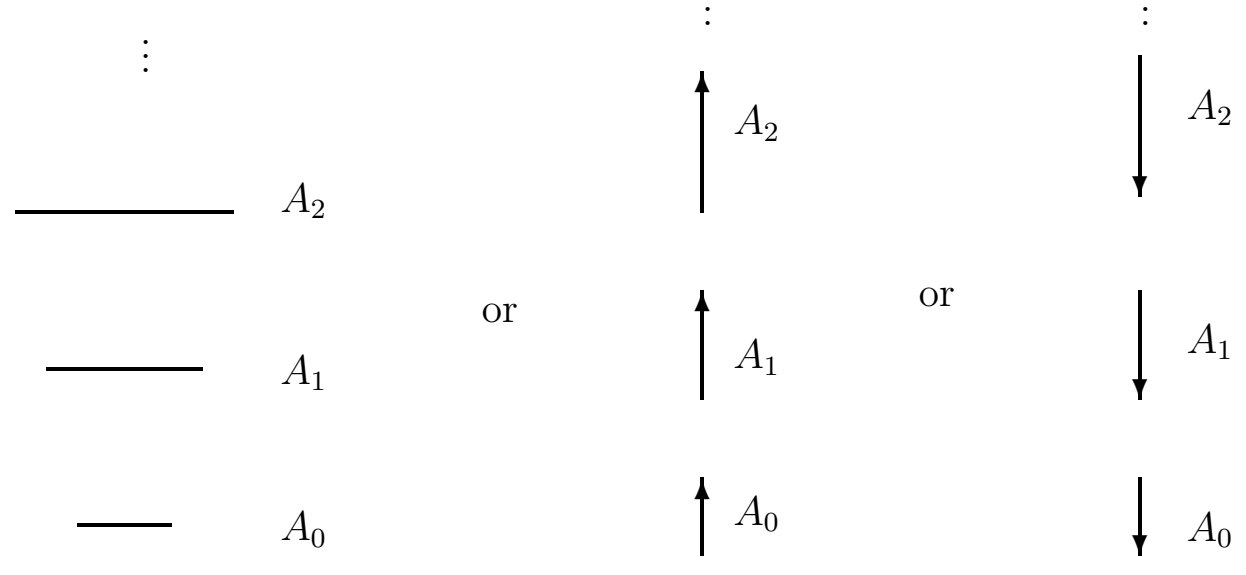

Figure 1

There are (at most) 9 possible types of our family $\left(A_{n}: n \in \omega\right)$ : Each $A_{n}$ can be well-ordered, co-well-ordered, or an antichain, and there are also 3 possible values for $c$. For example, if $c=$ " $<$ ", then set $A$ has one of the 3 forms given in the figure 1 .

However, our construction of a large family of incomparable functions will be "uniform", i.e., be the same in all cases. Note that if $c \in\{<\rangle$, and each $A_{n}$ is an antichain (leftmost possibility in the above picture), then $\bigcup_{n} A_{n}$ is not a complete partial order, since every element of $A_{n+1}$ is a minimal upper (or maximal lower) bound for any nontrivial subset of $A_{n}$.

For each $k$ we let $A_{2 k+1}^{\prime}$ be a singleton subset of $A_{2 k+1}$, and we let $A_{2 k}^{\prime}=A_{2 k}$. Now let $B:=\bigcup_{n} A_{n}^{\prime} \cup\{0,1\}$.

Step 3, case a. We are still assuming $\lambda=\aleph_{0}$. It is easy to see (by considering cases - one of them is sketched in figure 2) that the set $B$ defined in step 2 (case a) is a complete partial order. We leave the details to the reader.

Note that we still have $\prod_{n<\omega}\left|A_{n}^{\prime}\right|=\prod_{n \in \omega}\left|A_{2 n}^{\prime}\right|=\kappa^{\lambda}=2^{\kappa}$, since the cardinalities $\left(\left|A_{2 n}\right|: n \in \omega\right)$ are also increasing to $\kappa$. 


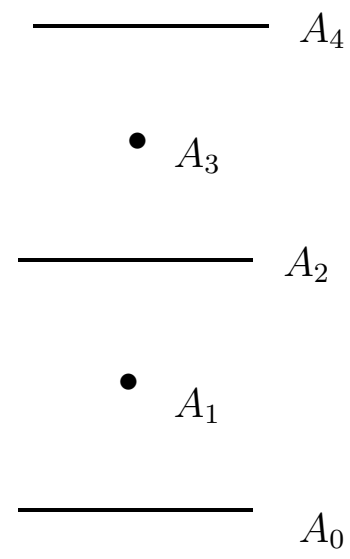

Figure 2

By 2.2 and the independence lemma 2.4, $\nu(L) \geq \nu(A)>2^{\kappa}$. This concludes the discussion of the case $\lambda=\aleph_{0}$.

Step 2, case b. Now we assume $\lambda>\aleph_{0}$. Note that now we are only aiming for $\kappa$ many pairwise incomparable monotone functions. Again let $F: \lambda \times \lambda \rightarrow\{<\rangle,, \|\}$ witness canonicity.

By dropping some of the sets $A_{\zeta}$ we may assume that the function $\zeta \mapsto F(0, \zeta)$ is constant, say with some value $c_{0}$. Thinning out three more times we may assume that there are constants $c_{0}, c_{1}, c_{2}, c_{3} \in$ $\{<\rangle,, \|\}$ such that

(a) $\forall \zeta>0, F(0, \zeta)=c_{0}$

(b) $\forall \zeta>1, F(1, \zeta)=c_{1}$

(c) $\forall \zeta>2, F(2, \zeta)=c_{2}$

(d) $\forall \zeta>3, F(3, \zeta)=c_{3}$

Choose $i_{0}<i_{1} \in\{0,1,2,3\}$ such that $c_{i_{0}}=c_{i_{1}}$, write $A_{*}$ for $A_{i_{0}}$, let $a_{*}$ be any element of $A_{i_{1}}$, and let $c_{*}=c_{i_{0}}$.

Depending on the value of $c_{*}$ we now have one the following possibilities: 
ORDER POLYNOMIALLY COMPLETE LATTICES MUST BE LARGE

(a) either every element of $A_{*}$ is incompatible with every element of any $A_{\zeta}, \zeta>4$,

(b) or we have for all $\zeta>4$ :

$$
\forall x \in A_{*} \forall y \in A_{\zeta}: x<a_{*}<y
$$

(c) or the dual of (b) is true.

Step 3, case b. Since all sets $A_{\zeta}$ are infinite, we have $\nu\left(A_{\zeta}\right)>\left|A_{\zeta}\right|$, so we can find pairwise incomparable monotone functions $\left(f_{\zeta, i}: i \in A_{\zeta}\right)$ from $A_{\zeta}$ to $A_{\zeta}$.

Let $f^{*}$ be the identity function on $\left\{a^{*}\right\}$, and let $\left(f_{*, \zeta}: 4<\zeta<\lambda\right)$ be a family of pairwise incomparable monotone functions from $A_{*}$ to $A_{*}$. (Recall that all our sets $A_{\zeta}$ had cardinality $\lambda$, so it is possible to find that many functions.)

Note that for $4<\zeta$ the family $\left(A_{*},\left\{a_{*}\right\}, A_{\zeta}\right)$ is canonical, so by the independence lemma we can conclude that $f_{*, \zeta} \cup f_{*} \cup f_{\zeta, i}$ is a monotone function. Moreover, $\left\{0,1, a_{*},\right\} \cup A_{*} \cup A_{\zeta}$ is a complete partial order (again we leave the easy task of checking this fact to the reader), so by 2.1 the function $f_{*, \zeta} \cup f_{*} \cup f_{\zeta, i}$ can be extended to a total monotone function $\hat{f}_{\zeta, i}: L \rightarrow L$.

Clearly any two functions $\hat{f}_{\zeta, i}, \hat{f}_{\zeta^{\prime}, i^{\prime}}$ are incomparable: If $\zeta \neq \zeta^{\prime}$ then this is due to the incomparability of $f_{*, \zeta}$ and $f_{*, \zeta^{\prime}}$, and for $\zeta=\zeta^{\prime}$ we use the incomparability of $f_{\zeta, i}$ and $f_{\zeta, i^{\prime}}$.

Note that the cardinality of the index set $\left\{(\zeta, i): 4<\zeta<\lambda, i \in A_{\zeta}\right\}$ is $\kappa$.

This concludes the discussion of the case $\lambda>\aleph_{0}$, and hence also the proof of the main lemma.

4.2. Conclusion. If $(L, \leq)$ is an o.p.c. lattice (or, slightly more generally, if $L$ is a partial order satisfying the conclusion of 3.9), then $\mu(L)=|L|$ is an inaccessible cardinal.

Proof. Let $\kappa=\mu(L)$. From 3.4 we know that $\kappa$ is a strong limit, and that $|L|=\kappa$. Assume that $\kappa$ is singular. 
First assume that $c f(\kappa)$ is uncountable. The main lemma tells us that $\nu(L)>\kappa$, so by 3.2 we conclude $\mu_{\infty}(L)>\kappa$, a contradiction.

Now we consider the second case: $c f(\kappa)=\aleph_{0}$. Here the main lemma tells us $\nu(L)>2^{\kappa}$. Since $2^{\kappa}$ has uncountable cofinality, we can again apply 3.2 and again get $\mu_{\infty}(L)>2^{\kappa}>\kappa$, a contradiction.

4.3. Remark. Note that the cardinality of an o.p.c. lattice cannot be a weakly compact cardinal.

Algebra und Diskrete Mathematik, Technische Universität Wien, Wiedner Hauptstrasse 8-10/118.2, A-1040 Wien, Austria

Current address: Mathematik WE 2, Freie Universität, Arnimallee 3, D-14195 Berlin, Germany

E-mail address: Martin.Goldstern@tuwien.ac.at

Department of Mathematics, Hebrew University of Jerusalem, GiVAT RAM, 91904 Jerusalem, ISRAel

E-mail address: shelah@math.huji.ac.il 